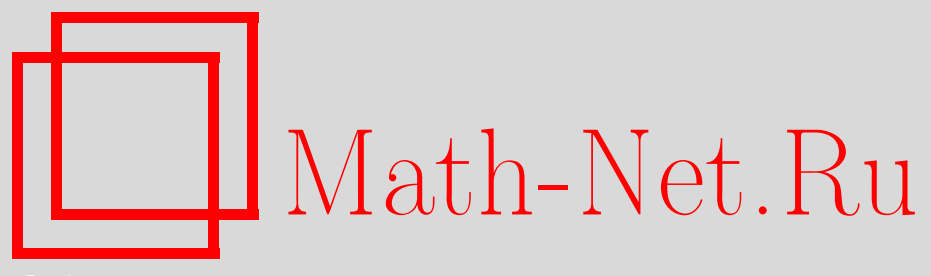

A. А. Лесных, Оценки решений дифференциальноразностных уравнений нейтрального типа, Матем. заметки, 2007, том 81, выпуск 4, 569-585

DOI: https://doi.org/10.4213/mzm3700

Использование Общероссийского математического портала Math-Net.Ru подразумевает, что вы прочитали и согласны с пользовательским соглашением http://www . mathnet.ru/rus/agreement

Параметры загрузки:

IP : 54.224 .187 .69

26 апреля 2023 г., 15:55:27

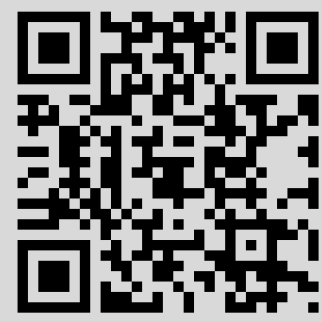


Том 81 выпуск 4 апрель 2007

УДК 517.929

\section{Оценки решений дифференциально-разностных уравнений нейтрального типа}

\section{А. А. Лесных}

В работе изучается скалярное дифференциально-разностное уравнение нейтрального типа общего вида

$$
\sum_{j=0}^{m} \int_{0}^{h} u^{(j)}(t-\theta) d \sigma_{j}(\theta)=0, \quad t>h,
$$

где $\sigma_{j}(\theta)$ - функции ограниченной вариации. Для решений этого уравнения получена оценка

$$
\|u(t)\|_{W_{2}^{m}(T, T+h)} \leqslant C T^{q-1} e^{\varkappa T}\|u(t)\|_{W_{2}^{m}(0, h)},
$$

где $C$ - постоянная, не зависящая от $u_{0}(t)$, а значения величин $q$ и $\varkappa$ определяются свойствами характеристического определителя этого уравнения. Ранее эта оценка доказывалась для уравнений менее общего вида. Например, для кусочно постоянных функций $\sigma_{j}(\theta)$, или для случая, когда $\sigma_{m}(\theta)$ имеет скачки в обеих точках $\theta=0$ и $\theta=h$. В настоящей работе эта оценка получена только при условии, что $\sigma_{m}(\theta)$ имеет скачок в точке $\theta=0$, которое необходимо для корректной разрешимости начальной задачи.

Библиография: 19 названий.

1. Введение. Одним из важных вопросов в теории функционально-дифференциальных уравнений является вопрос об асимптотическом поведении решений. Среди обширной литературы по этому вопросу отметим монографии [1]-[5], в которых могут быть найдены дальнейшие ссылки.

В этой работе мы получаем точные оценки решений уравнений нейтрального типа достаточно общего вида. Отметим следующие результаты, наиболее близкие к результатам нашей работы. В [6] изучалась начальная задача

$$
\begin{gathered}
\frac{d}{d t}\left(u(t)+\int_{0}^{h} u(t-\theta) d \mu(\theta)\right)+\int_{0}^{h} u(t-\theta) d \eta(\theta)=0, \quad t>h, \\
u(t)=u_{0}(t), \quad t \in[0, h]
\end{gathered}
$$

Работа выполнена при поддержке Российского фонда фундаментальных исследований (грант № 04-01-00712) и программы "Ведущие научные школы" (грант № НШ-5247.2006.1).

(C) А. А. Лесных, 2007 
где $\mu(\theta), \eta(\theta)$ - функции ограниченной вариации. Для решений этой задачи при предположении, что $\mu(\theta)$ непрерывна при $\theta=0$, получена следующая оценка в норме пространства непрерывных функций:

$$
\|u\|_{C[t, t+h]} \leqslant d e^{(\varkappa+\varepsilon) t}\left\|u_{0}\right\|_{C[0, h]} .
$$

В этой оценке число $\varepsilon>0$ в показателе экспоненты можно взять сколь угодно малым. В этом смысле указанная оценка является неточной.

Неточные оценки такого типа давно и хорошо известны (см. указанные выше монографии). Однако точные оценки решений уравнений нейтрального типа различных видов были получены сравнительно недавно. Наиболее общими, на наш взгляд, являются следующие результаты. В [7] изучалось уравнение вида

$$
\sum_{k=0}^{n} \sum_{j=0}^{m} A_{k j} u^{(j)}\left(t-h_{k}\right)=f(t), \quad t>h,
$$

где $A_{k j}-$ постоянные матрицы, $\operatorname{det} A_{0 m} \neq 0$. Для решений этого уравнения получена оценка, которая при $f(t) \equiv 0$ имеет вид

$$
\|u\|_{W_{2}^{m}(t-h, t)} \leqslant C(t+1)^{q-1} e^{\varkappa t}\left\|u_{0}\right\|_{W_{2}^{m}(0, h)}, \quad t \geqslant h,
$$

где постоянная $C$ не зависит от функции $u_{0}(t)$, а $q$ и $\varkappa$ - некоторые действительные числа. Здесь уже нельзя уменьшить число $\varkappa$ и заменить его на $\varkappa-\varepsilon$, т.е. эта оценка является точной.

В уравнении (3) участвует лишь конечное число запаздываний по времени, задаваемых числами $h_{k}$. В то же время интерес вызывают такие уравнения, запаздывания в которых заданы в более общем виде. Например, в уравнении (1) запаздывания и коэффициенты задаются функциями ограниченной вариации $\mu(\theta)$ и $\eta(\theta)$. В случае, когда эти функции кусочно-постоянные с конечным числом скачков, мы получаем уравнение с конечным числом запаздываний. Точная оценка решений нейтральных уравнений вида (1), запаздывания и коэффициенты в которых задаются функциями ограниченной вариации, получена в [8], где изучается векторное уравнение вида

$$
\frac{d}{d t} \int_{0}^{h} u(t-\theta) d \mu(\theta)+\int_{0}^{h} u(t-\theta) \eta(\theta) d \theta=f(t),
$$

элементы матриц $\mu(\theta)$ лежат в $B V[0, h]$, а элементы матриц $\eta(\theta)$ лежат в $L_{2}(0, h)$. Однако оценка решений получена при более жестких ограничениях на коэффициенты. А именно, предполагается, что функция $\mu(\theta)$ имеет скачок не только при $\theta=0$, но еще и при $\theta=h$.

В этой работе мы изучаем дифференциально-разностное уравнение нейтрального типа, в котором сдвиги по времени и коэффициенты задаются функциями ограниченной вариации по аналогии с [6] и [8], а на коэффициенты наложены минимальные ограничения, гарантирующие лишь корректную разрешимость начальной задачи. Для решений соответствующей начальной задачи получена точная оценка в пространстве $W_{2}^{m}$. Сформулируем основной результат работы. 
Рассмотрим следующую начальную задачу для скалярного дифференциально-разностного уравнения нейтрального типа:

$$
\begin{array}{r}
\sum_{j=0}^{m} \int_{0}^{h} u^{(j)}(t-\theta) d \sigma_{j}(\theta)=0, \quad t>h, \\
u(t)=u_{0}(t), \quad t \in[0, h],
\end{array}
$$

где функции $\sigma_{j}(\theta) \in B V[0, h]$. Целая функция

$$
\Delta(\lambda)=\sum_{j=0}^{m} \lambda^{j} \int_{0}^{h} e^{-\lambda \theta} d \sigma_{j}(\theta)
$$

называется характеристическим определителем уравнения (5).

Для точной формулировки основного результата работы нам понадобится следующее утверждение.

Теорема 1. Пусть $\sigma_{m}(0+)-\sigma_{m}(0) \neq 0$. Тогда все нули $\Delta(\lambda)$ лежат в некоторой левой полуплоскости $\{\operatorname{Re} \lambda \leqslant C\}$, и поэтому существует иисло

$$
\varkappa=\sup \{\operatorname{Re} \lambda: \Delta(\lambda)=0\}<\infty .
$$

Кроме того, число нулей $n_{\Delta}(P(a, b, h))$ определителя $\Delta(\lambda)$, лежащих в прямоугольнике

$$
P(a, b, h)=\{\lambda \in \mathbb{C}: \operatorname{Re} \lambda \in(a, \varkappa), \operatorname{Im} \lambda \in(b, b+h)\},
$$

не зависит от $b \in \mathbb{R}$ и существует предел

$$
q=\lim _{a \rightarrow \varkappa} \lim _{h \rightarrow 0} \max _{b \in \mathbb{R}} n_{\Delta}(P(a, b, h)) .
$$

Основной результат работы содержится в следующей теореме.

Теорема 2. Пусть $\sigma_{m}(0+)-\sigma_{m}(0) \neq 0$. Тогда при достаточно болъиих $T>0$ для решения и(t) задачи (5),(6) имеет место оценка

$$
\|u\|_{W_{2}^{m}(T, T+h)} \leqslant C T^{q-1} e^{\varkappa T}\left\|u_{0}\right\|_{W_{2}^{m}(0, h)},
$$

где числа и и q определены в теореме 1 , а постоянная $C$ не зависит от начальной бункции $u_{0}(t)$.

В данной работе используется операционный подход (см., например, [2]), основанный на представлении решения задачи (5), (6) в виде преобразования Лапласа. При таком подходе ключевую роль играют вопросы о распределении нулей характеристического определителя $\Delta(\lambda)$ и его оценки на контурах интегрирования. Эти вопросы достаточно хорошо изучены (см., например, [2], [9]-[11]) в случае, когда $\Delta(\lambda)$ есть квазиполином вида

$$
g(\lambda)=\sum_{j=0}^{n} p_{j} \lambda^{m_{j}} e^{\beta_{j} \lambda},
$$

где $p_{j}, m_{j}, \beta_{j}$ - некоторые числа. Характеристический определитель $\Delta(\lambda)$, соответствующий уравнению (5), имеет более общий вид (7), для которого развитая ранее 
техника не применима. Мы предлагаем новый подход для получения оценок таких функций, основанный на технике работы [12]. Мы также существенно используем методы работы [13] при оценке интегралов по счетному числу контуров. Результаты и методы нашей работы могут быть без существенных изменений перенесены на случай векторных и неоднородных уравнений нейтрального типа.

На протяжении работы числа $C, C_{0}, C_{1}, \ldots$ обозначают постоянные, возможно, разные в разных формулах. Через $n_{f}(G)$ обозначается число нулей функции $f$ в области $G$, а через $M_{f}(G)$ - максимум модуля функции $f$ в области $G$. Кроме того, помимо обычной метрики мы будем использовать в комплексной плоскости метрику

$$
\widetilde{\rho}\left(z_{1}, z_{2}\right)=\max \left\{\left|\operatorname{Re}\left(z_{1}-z_{2}\right)\right|,\left|\operatorname{Im}\left(z_{1}-z_{2}\right)\right|\right\}
$$

Работа построена следующим образом. В п. 2 устанавливаются используемые в дальнейшем свойства характеристического определителя и его нулей. В п. 3 решение задачи (5), (6) записывается в виде преобразования Лапласа и строятся контуры интегрирования. Пункт 4 посвящен оценке интегралов по построенным контурам и завершению доказательства теоремы 2.

2. Свойства характеристического определителя и его нулей. В данном пункте мы приводим необходимые в дальнейшем свойства характеристического определителя и его нулей.

Лемма 1. Пусть $\sigma_{m}(0+)-\sigma_{m}(0) \neq 0$. Тогда характеристический определитель $\Delta(\lambda)$ обладает следующими свойствами:

а) для достаточно больших $\operatorname{Re} \lambda$ имеется асимптотика

$$
\Delta(\lambda) \asymp \lambda^{m}
$$

b) в любой полуплоскости $\left\{\operatorname{Re} \lambda>C_{1}\right\}$ для характеристического определителя и его производных справедливы оченки

$$
\left|\Delta^{(j)}(\lambda)\right|<C_{2}(|\lambda|+1)^{m}, \quad j=0,1,2, \ldots, m
$$

с) для всех $\varepsilon \in(0,1 / 2]$ в любой полуплоскости $\left\{\operatorname{Re} \lambda>C_{1}\right\}$ вне $\varepsilon$-окрестности нулей $\Delta(\lambda)$ для достаточно больших $\lambda$ справедлива оценка

$$
|\Delta(\lambda)|>C_{2}|\lambda|^{m}
$$

где постоянная $C_{2}$ зависит только от в и $C_{1}$.

ДокАЗАтельство. Запишем $\Delta(\lambda)$ в виде

$$
\begin{gathered}
\Delta(\lambda)=\Delta_{1}(\lambda)+\Delta_{2}(\lambda)+\Delta_{3}(\lambda) \\
\Delta_{1}(\lambda)=\lambda^{m}\left(\sigma_{m}(0+)-\sigma_{m}(0)\right), \quad \Delta_{2}(\lambda)=\lambda^{m} \int_{0}^{h} e^{-\lambda \theta} d \widetilde{\sigma}_{m}(\theta), \\
\Delta_{3}(\lambda)=\sum_{j=0}^{m-1} \lambda^{j} \int_{0}^{h} e^{-\lambda \theta} d \sigma_{j}(\theta)
\end{gathered}
$$


где $\widetilde{\sigma}_{m}(\theta)=\sigma_{m}(\theta)$ при $\theta \in(0, h]$ и $\widetilde{\sigma}_{m}(\theta)$ непрерывна в нуле. Пользуясь непрерывностью функции $\widetilde{\sigma}_{m}(\theta)$ в нуле, можно показать, что

$$
\int_{0}^{h} e^{-\theta N}\left|d \widetilde{\sigma}_{m}(\theta)\right| \rightarrow 0 \quad \text { при } \quad N \rightarrow+\infty,
$$

откуда получаем в области $\{\operatorname{Re} \lambda>C\}$ представление

$$
\Delta_{2}(\lambda)=o(1) \lambda^{m}
$$

где $o(1) \rightarrow 0$ при $\operatorname{Re} \lambda \rightarrow+\infty$. Далее, в области $\{\operatorname{Re} \lambda>C\}$ при $j=0,1, \ldots, m-1$ имеем

$$
\left|\lambda^{j} \int_{0}^{h} e^{-\lambda \theta} d \sigma_{j}(\theta)\right| \leqslant|\lambda|^{j} \int_{0}^{h} e^{-\theta \operatorname{Re} \lambda}\left|d \sigma_{j}(\theta)\right| \leqslant C|\lambda|^{j} .
$$

Из (12) и (13) следует, что для достаточно больших $\operatorname{Re} \lambda$ выполняются оценки $\left|\Delta_{2}(\lambda)\right|<\left|\Delta_{1}(\lambda)\right| / 3$ и $\left|\Delta_{3}(\lambda)\right|<\left|\Delta_{1}(\lambda)\right| / 3$. Отсюда получаем утверждение а) леммы.

Оценка сверху $|\Delta(\lambda)|<C_{1}(|\lambda|+1)^{m}$ справедлива не только при достаточно больших $\operatorname{Re} \lambda$, но и в любой полуплоскости $\left\{\operatorname{Re} \lambda>C_{2}\right\}$, так как там $\left|\Delta_{k}(\lambda)\right|<C_{3}|\lambda|^{m}$, $k=1,2,3$, в виду (12) и (13). Аналогично можно получить оценки производных $\Delta^{(j)}(\lambda)$ и тем самым доказать утверждение b) леммы.

Докажем теперь утверждение с). Мы уже получили асимптотику (10) для достаточно больших $\operatorname{Re} \lambda$, поэтому достаточно доказать оценку (11) в полосе $\{\operatorname{Re} \lambda \in$ $\left.\left(a_{1}, a_{2}\right)\right\}$ для любых $a_{1}, a_{2}$. Введем функцию $f(z)=\Delta(z+i b)$, область

$$
S=\left\{z \in \mathbb{C}: \operatorname{Re} z \in\left(a_{1}, a_{2}\right), \operatorname{Im} z \in(0, h)\right\}
$$

и $\Omega=\{z \in \mathbb{C}: \widetilde{\rho}(z, S)<\delta\}-\delta$-окрестность области $S$ в метрике $\widetilde{\rho}$, где $b, h$, $\delta$ - некоторые действительные числа. Применим к функции $f(z)$ и областям $\Omega$ и $S$ лемму 2.1 из [12]. Получим, что вне некоторого исключительного множества кружков с суммой радиусов меньше $\varepsilon$ выполняется оценка

$$
\ln |f(z)| \geqslant C \ln \varepsilon\left(\ln M_{f}(\Omega)-\ln M_{f}(S)\right)+\ln M_{f}(S),
$$

где постоянная $C$ не зависит от функции $f(z)$. Без ограничения общности, точка $a_{2}+i b-$ это самый удаленный от нуля угол области $S$. Тогда из полученных выше оценок имеем для достаточно большого числа $a_{2}$ неравенства

$$
\begin{aligned}
& M_{f}(S) \geqslant\left|\Delta\left(a_{2}+i(b+h)\right)\right| \geqslant C_{1}\left|a_{2}+i(b+h)\right|^{m}, \\
& M_{f}(\Omega) \leqslant C_{2}\left|a_{2}+\delta+i(b+h+\delta)\right|^{m} .
\end{aligned}
$$

Подставляя эти неравенства в (14), получаем

$$
\ln |f(z)| \geqslant \ln \left(C\left|a_{2}+i(b+h)\right|^{m}\right)
$$

где постоянная $C$ не зависит от выбора числа $b$. Вспоминая определение функции $f(z)$, получаем искомую оценку для характеристического определителя $\Delta(\lambda)$.

Для доказательства теоремы 1 нам понадобится следующая лемма об оценке числа нулей аналитической функции. 
Лемма 2. Пусть $f(z)$ - функиия, аналитическая в односвязной области $\Omega$ и некоторой ее окрестности, $S$ - компакт, $S \subset \Omega$. Тогда

$$
n_{f}(S) \leqslant C\left(\ln M_{f}(\Omega)-\ln M_{f}(S)\right),
$$

где постоянная $C$ зависит от $\Omega$ и $S$, но не зависит от функции $f(z)$.

ДокАЗАТЕЛЬСтво. Для случая круговых областей аналогичная оценка известна (см. [9; гл. 1, §5, лемма 4]). Чтобы доказать эту оценку в общем случае, воспользуемся приемом из [12; с. 324]. Пусть $\psi_{\mu}(z)$ - конформное отображение круга $K_{R}=\{|z|<R\}$ на область $\Omega$ такое, что $\psi_{\mu}(0)=\mu \in S$. Пусть $r<R$ - наименьшее число такое, что $S \subset \psi_{\mu}\left(K_{r}\right)$ для всех $\mu \in S$. Положим $\mu_{0} \in S$ таким, что $f\left(\mu_{0}\right)=M_{f}(S)$. Рассмотрим функцию $F(z)=f\left(\psi_{\mu_{0}}(z)\right)$. Так как $n_{F}\left(K_{t}\right)$ не убывает по $t \in[r, R]$, то

$$
\int_{r}^{R} \frac{n_{F}\left(K_{t}\right)}{t} d t \geqslant n_{F}\left(K_{r}\right) \int_{r}^{R} \frac{d t}{t}=C n_{F}\left(K_{r}\right) .
$$

Из этой оценки получаем

$$
n_{f}(S)=n_{F}\left(K_{r}\right) \leqslant C \int_{r}^{R} \frac{n_{F}\left(K_{t}\right)}{t} d t \leqslant C \int_{0}^{R} \frac{n_{F}\left(K_{t}\right)}{t} d t .
$$

Применив теорему Иенсена [9; гл. $1, \S 5]$, продолжим цепочку неравенств

$$
\begin{aligned}
n_{f}(S) & \leqslant C\left(\frac{1}{2 \pi} \int_{0}^{2 \pi} \ln \left|F\left(R e^{i \theta}\right)\right| d \theta-\ln |F(0)|\right) \\
& \leqslant C\left(\ln M_{f}\left(K_{R}\right)-\ln |F(0)|\right)=C\left(\ln M_{f}\left(K_{R}\right)-\ln M_{f}(S)\right) .
\end{aligned}
$$

Лемма доказана.

ДоКАЗАТЕЛЬСТво тЕОРЕМЫ 1 . Существование указанного числа $\varkappa$ следует из п. а) леммы 1. Докажем существование предела (8). В случае, когда функции $\sigma_{j}(\theta)$ имеют конечное число скачков, это доказано в [14]. Возьмем достаточно большое число $a_{2}>\varkappa$, положим $a_{1}=a$ и введем функцию $f(z)$ и области $S$ и $\Omega$ так же, как и при доказательстве леммы 1. Применяя лемму 2 и оценки $(15),(16)$, получим, что число $n_{\Delta}(P(a, b, h))$ ограничено сверху постоянной, не зависящей от $b$. Тогда существует функция $\max _{b \in \mathbb{R}} n_{\Delta}(P(a, b, h))$ аргументов $a$ и $h$. Эта функция не возрастает при $a \nearrow \varkappa$ и $h \searrow 0$ и ограничена снизу, поэтому существует предел (8).

3. Запись решения и построение контуров интегрирования. Мы будем оценивать решение $u(t)$ задачи $(5),(6)$ следующим образом. Сначала решение записывается в виде обратного преобразования Лапласа, которое представляет собой интеграл по некоторой вертикальной прямой $b+i \mathbb{R}$, где $b>\varkappa$. Затем интеграл по $b+i \mathbb{R}$ представляется в виде суммы интегралов по некоторой ломаной Г и некоторым контурам $\partial G_{k}, k=1,2, \ldots$. Ломаная $\Gamma$ находится уже слева от прямой $\varkappa+i \mathbb{R}$ достаточно близко к некоторой прямой $a+i \mathbb{R}, a<\varkappa$, а интеграл по этой ломаной не вносит существенного вклада в оценку решения $u(t)$. Основной вклад в оценку решения вносят интегралы по контурам $\partial G_{k}$. Эти контуры окружают нули характеристического определителя $\Delta(\lambda)$, находящиеся в вертикальной полосе между $Г$ и $b+i \mathbb{R}$. Ключевую роль при получении оценок интегралов играет тот факт, что 
расстояние от контуров интегрирования $\Gamma$ и $\partial G_{k}$ до нулей $\Delta(\lambda)$ ограничено снизу. Это позволяет с помощью леммы 1 оценить сверху $\Delta^{-1}(\lambda)$ и соответствующие интегралы.

Итак, приступим к реализации намеченного плана. Применяя к уравнению (5) преобразование Лапласа, решение $u(t)$ задачи $(5),(6)$ можно записать в виде

$$
u(t)=\lim _{n \rightarrow \infty} \int_{b+i d_{-n}}^{b+i d_{n}} e^{t \lambda} \Delta^{-1}(\lambda) \sum_{j=0}^{m} \int_{0}^{h} p_{j}(\lambda, \theta) d \sigma_{j}(\theta) d \lambda,
$$

где $b \in \mathbb{R}$ - достаточно большое число, $d_{n} \rightarrow \pm \infty$ при $n \rightarrow \pm \infty$,

$$
\begin{aligned}
p_{j}(\lambda, \theta)=e^{-\lambda \theta} & \left(u_{0}^{(j-1)}(0)+\lambda u_{0}^{(j-2)}(0)+\cdots+\lambda^{j-1} u_{0}(0)\right) \\
& +\int_{0}^{h-\theta} u_{0}^{(j)}(\tau) e^{-\lambda(\tau+\theta)} d \tau, \quad j=0,1, \ldots, m .
\end{aligned}
$$

Предположим сначала для простоты, что

$$
u_{0}(0)=u_{0}^{\prime}(0)=\cdots=u_{0}^{(m-1)}(0)=0 .
$$

Тогда

$$
p_{j}(\lambda, \theta)=\int_{0}^{h-\theta} u_{0}^{(j)}(\tau) e^{-\lambda(\tau+\theta)} d \tau, \quad j=0,1, \ldots, m .
$$

Так как функция $n_{\Delta}(P(a, b, h))$ принимает целые значения и не возрастает при $a \nearrow \varkappa$ и $h \searrow 0$, то найдутся такие числа $a, \delta, h$, что $q=\max _{d \in \mathbb{R}} n_{\Delta}(P(a-\delta, b, h))$ и $a+\delta<\varkappa$.

Построим сначала ломаную $\Gamma$, расположенную достаточно близко к прямой $a+i \mathbb{R}$, точнее, $\Gamma \subset \Pi_{\delta}=\{\operatorname{Re} \lambda \in[a-\delta, a+\delta]\}$. Причем отрезки этой ломаной будут лежать на сетке

$$
S_{\varepsilon}=\bigcup_{k \in \mathbb{Z}}(\{\operatorname{Re} \lambda=a+k \varepsilon\} \cup\{\operatorname{Im} \lambda=k \varepsilon\}) .
$$

Обозначим $\Lambda_{\varepsilon}-\varepsilon$-окрестность нулей $\Delta(\lambda)$.

Лемма 3. Пусть $a, b, \delta, h$ - числа, определенные выше. Тогда для достаточно малого числа $\varepsilon>0$ найдутся числа $d_{n} \in \mathbb{R}, n \in \mathbb{Z}$, такие, что $h / 2 \leqslant d_{n+1}-d_{n} \leqslant h$ $u \tau_{n} \in S_{\varepsilon} \backslash \Lambda_{\varepsilon}$, где $\tau_{n}=\left[a+i d_{n}, b+i d_{n}\right]$. Найдутся также ломаные

$$
\gamma_{n} \in\left(\Pi_{\delta} \cap\left\{\operatorname{Im} \lambda \in\left[d_{n}, d_{n+1}\right]\right\} \cap S_{\varepsilon}\right) \backslash \Lambda_{\varepsilon}, \quad n \in \mathbb{Z},
$$

с концами $a+i d_{n}$ и $a+i d_{n+1}$ такие, что их длины ограничены равномерно по $n \in \mathbb{Z}$.

ДокАЗАТЕЛЬство. Построим вокруг каждого нуля характеристического определителя $\Delta(\lambda)$ такой прямоугольник, что его стороны лежат на сетке $S_{\varepsilon}$ и расстояние от его сторон до нуля $\Delta(\lambda)$ лежит в интервале $[\varepsilon, 2 \varepsilon)$. Объединение таких прямоугольников для всех нулей $\Delta(\lambda)$ состоит из некоторых связных компонент. Мы будем рассматривать те связные компоненты, которые либо лежат целиком справа от прямой $a+i \mathbb{R}$, либо пересекают ее. Объединение таких связных компонент обозначим через $\Omega_{\varepsilon}$. В виду выбора чисел $a, \delta, h$ каждая связная компонента множества $\Omega_{\varepsilon}$ содержит не более $q$ нулей $\Delta(\lambda)$ и имеет в метрике $\widetilde{\rho}$ диаметр не больше $4 \varepsilon q$. 
Построим числа $d_{n}$. Пусть число $\varepsilon>0$ настолько мало, что $4 \varepsilon q<h / 2$. Тогда множество $\operatorname{Im} \Omega_{\varepsilon}$ состоит из отрезков с длиной меньше $h / 2$. Число $d_{0}$ выберем произвольным образом из множества $\operatorname{Im} S_{\varepsilon} \backslash \operatorname{Im} \Omega_{\varepsilon}$ (это множество не пусто, так как $\varepsilon<h / 2)$. Числа $d_{1}, d_{2}, \ldots$ определим рекуррентно следующим образом:

$$
d_{n}=\sup \left\{\left(\left(-\infty, d_{n-1}+h\right] \cap \operatorname{Im} S_{\varepsilon}\right) \backslash \operatorname{Im} \Omega_{\varepsilon}\right\}, \quad n=1,2, \ldots
$$

Так как множество $\operatorname{Im} \Omega_{\varepsilon}$ состоит из отрезков с длиной меньше $h / 2$, то $d_{n} \in\left[d_{n-1}+\right.$ $\left.h / 2, d_{n-1}+h\right] \cap \operatorname{Im} S_{\varepsilon}$ (это множество не пусто, так как $\varepsilon<h / 2$ ), и поэтому, $h / 2 \leqslant$ $d_{n}-d_{n-1} \leqslant h$. Числа $d_{-1}, d_{-2}, \ldots$ определяются аналогичным образом:

$$
d_{n}=\inf \left\{\left(\left[d_{-(n-1)}-h,+\infty\right) \cap \operatorname{Im} S_{\varepsilon}\right) \backslash \operatorname{Im} \Omega_{\varepsilon}\right\}, \quad n=-1,-2, \ldots .
$$

Построим теперь ломаные $\gamma_{n}$. Пусть число $\varepsilon>0$ настолько мало, что $4 \varepsilon q<\delta / 2$. Тогда каждая связная компонента множества $\Omega_{\varepsilon}$ имеет в метрике $\widetilde{\rho}$ диаметр, не превосходящий $\delta / 2$. Обозначим $\rho_{n}=\left[a+i d_{n}, a+i d_{n+1}\right]$. Если $\rho_{n} \cap \Omega_{\varepsilon}=\varnothing$, то положим $\gamma_{n}=\rho_{n}$. Иначе $\rho_{n} \cap \Omega_{\varepsilon}$ представляет собой объединение некоторых вертикальных отрезков $\rho_{n k}$, число которых не превосходит $q$ для каждого $n$. Заменим каждый отрезок $\rho_{n k}$ некоторой ломаной $\gamma_{n k}^{\prime}$, т.е. положим

$$
\gamma_{n}=\left(\rho_{n} \backslash \Omega_{\varepsilon}\right) \cup \bigcup_{k} \gamma_{n k}^{\prime},
$$

где $\gamma_{n k}^{\prime}$ - это участок границы множества $\Omega_{\varepsilon}$, соединяющий концы отрезка $\rho_{n k}$. Так как каждая связная компонента множества $\Omega_{\varepsilon}$, лежащая в $\Pi_{\delta}$, имеет в метрике $\widetilde{\rho}$ диаметр, не превосходящий $\delta / 2$, то $\gamma_{n k}^{\prime} \subset \Pi_{\delta}$. Длина отрезка $\rho_{n}$ не превосходит $h$, длина ломаных $\gamma_{n k}^{\prime}$ не превосходит $4 \varepsilon \cdot 4 q=16 \varepsilon q$, поэтому длина ломаной $\gamma_{n}$ не превосходит $h+16 \varepsilon q^{2} \leqslant C$, равномерно по $n$.

В соответствии с этой леммой, положим $\Gamma=\bigcup \gamma_{k}$ и заменим в (21) интеграл по отрезку $\left[b+i d_{-n}, b+i d_{n}\right]$ на сумму интегралов по отрезкам $\tau_{-n}, \tau_{n}$, ломаным $\gamma_{k}$, лежащим между $\tau_{-n}$ и $\tau_{n}$, и сумму по некоторым контурам, окружающим те полюса подынтегральной функции (т.е. нули $\Delta(\lambda)$ ), которые попали между $\Gamma$ и $b+i \mathbb{R}$ и между $\tau_{-n}$ и $\tau_{n}$.

В качестве контуров, окружающих полюса, возьмем следующие контуры $\partial G_{k}$. Обозначим через П вертикальную полосу, ограниченную ломаной $Г$ и прямой $b+i \mathbb{R}$. Положим $G_{k}, k=1,2, \ldots,-$ связные компоненты множества $\Lambda_{\varepsilon} \cap \Pi$, а $\partial G_{k}-$ их граница. Заметим, что в виду выбора чисел $a$ и $h$, ломаной $\Gamma$ и отрезков $\tau_{n}$, области $G_{k}$ не пересекаются с $\Gamma$ и $\tau_{n}$, и число нулей $\Delta(\lambda)$, лежащих в полосе П между отрезками $\tau_{n}$ и $\tau_{n+1}$ (а значит, и число нулей в каждой области $G_{k}$ ) не превосходит $q$.

4. Оценка интегралов. Запишем решение $u(t)$ в виде

$$
u(t)=\lim _{n \rightarrow \infty} \int_{b+i d_{-n}}^{b+i d_{n}} U(t, \lambda) d \lambda,
$$

где

$$
U(t, \lambda)=e^{t \lambda} \Delta^{-1}(\lambda) \sum_{j=0}^{m} \int_{0}^{h} p_{j}(\lambda, \theta) d \sigma_{j}(\theta) .
$$


Интеграл по отрезку $\left[b+i d_{-n}, b+i d_{n}\right]$ представим в виде следующей суммы:

$$
\int_{b+i d_{-n}}^{b+i d_{n}}=\int_{\tau_{-n}}+\int_{\tau_{n}}+\sum_{k=-n}^{n-1} \int_{\gamma_{n}}+\sum_{G_{k} \subset \Pi_{n}} \int_{\partial G_{k}},
$$

где $\Pi_{n}-$ часть полосы П, лежащая между $\tau_{-n}$ и $\tau_{n}$. Далее, будем последовательно оценивать интегралы в правой части $(25)$ в пространстве $W_{2}^{m}(T, T+h)$ при $n \rightarrow \infty$.

Оценим сначала интеграл по ломаной Г.

Лемма 4. При всех $T>0$ справедлива оценка

$$
\left\|\int_{\Gamma} U(t, \lambda) d \lambda\right\|_{W_{2}^{m}(T, T+h)} \leqslant C e^{(a+\delta) T}\left\|u_{0}(t)\right\|_{W_{2}^{m}(0, h)},
$$

где постоянная $C$ не зависит от начальной функции $u_{0}(t)$.

ДокАЗАтЕЛЬСтво. Фиксируем порядок производной $l \in\{0,1, \ldots, m\}$ и оценим норму функции

$$
v_{n}(t)=\frac{d^{l}}{d t^{l}} \int_{\Gamma_{n}} U(t, \lambda) d \lambda
$$

в пространстве $L_{2}(T, T+h)$. Запишем функцию $v_{n}(t)$ в виде

$$
v_{n}(t)=\sum_{j=0}^{m} \int_{0}^{h} d \sigma_{j}(\theta) \int_{\Gamma_{n}} e^{t \lambda} f(\lambda) d \lambda
$$

где

$$
f(\lambda)=\lambda^{l} \Delta^{-1}(\lambda) e^{-\lambda \theta} g(\lambda), \quad g(\lambda)=\int_{0}^{h-\theta} u_{0}^{(j)}(\tau) e^{-\lambda \tau} d \tau .
$$

Ломаная $\Gamma$ состоит из отрезков сетки $S_{\varepsilon}$. Будем отдельно оценивать сумму интегралов по вертикальным и горизонтальным отрезкам. Заметим, что ломаная $Г$ лежит в полосе $\Pi_{\delta}$, поэтому все вертикальные отрезки этой ломаной лежат на конечном числе вертикальных прямых $a+k \varepsilon+i \mathbb{R}$, где целое число $k \in(-\delta / \varepsilon, \delta / \varepsilon)$.

Введем на $S_{\varepsilon}$ функцию

$$
\widetilde{f}(\lambda)= \begin{cases}f(\lambda), & \lambda \in \Gamma, \\ 0, & \lambda \notin \Gamma,\end{cases}
$$

и запишем интеграл по ломаной Г в виде суммы

$$
\int_{\Gamma} e^{t \lambda} f(\lambda) d \lambda=\sum_{k=\lceil-\delta / \varepsilon\rceil}^{\lfloor\delta / \varepsilon\rfloor} \int_{a+k \varepsilon+i \mathbb{R}} e^{t \lambda} \tilde{f}(\lambda) d \lambda+\sum_{n \in \mathbb{Z}} \int_{l_{n}} e^{t \lambda} \widetilde{f}(\lambda) d \lambda,
$$

где $l_{n}=[a-\delta+i n \varepsilon, a+\delta+i n \varepsilon]$. Оценим сначала сумму интегралов по вертикальным отрезкам ломаной $\Gamma$, т.е. первую сумму из $(26)$ в пространстве $L_{2}(T, T+h)$. Так как функция $\lambda^{l} \Delta^{-1}(\lambda) e^{-\lambda \theta}$ ограничена на $\Gamma$, то $\widetilde{f}(\lambda) \in L_{2}(k \varepsilon+i \mathbb{R})$ при всех целых $k$ таких, что $k \varepsilon \in(a-\delta, a+\delta)$. Тогда для всех таких $k$ имеем цепочку неравенств

$$
\begin{gathered}
\left\|\int_{a+k \varepsilon+i \mathbb{R}} e^{t \lambda} \tilde{f}(\lambda) d \lambda\right\|_{L_{2}(T, T+h)}=\left\|\int_{-\infty}^{+\infty} e^{(a+k \varepsilon) t} e^{i \xi \lambda} \tilde{f}(a+k \varepsilon+i \xi) d \lambda\right\|_{L_{2}(T, T+h)} \\
\leqslant C e^{(a+\delta) T}\left\|\int_{-\infty}^{+\infty} e^{i \xi \lambda} \tilde{f}(a+k \varepsilon+i \xi) d \lambda\right\|_{L_{2}(T, T+h)} \leqslant C e^{(a+\delta) T}\|\tilde{f}(\lambda)\|_{L_{2}(a+k \varepsilon+i \mathbb{R})} .
\end{gathered}
$$

Здесь в последнем неравенстве использована теорема Планшереля [15; с. 504]. 
Оценим теперь сумму интегралов по горизонтальным отрезкам ломаной Г, т.е. вторую сумму из $(26)$ в пространстве $L_{2}(T, T+h)$. Введем функцию

$$
F_{N}(t)=\sum_{n=-N}^{N} \int_{l_{n}} e^{t \lambda} \widetilde{f}(\lambda) d \lambda
$$

возьмем произвольную функцию $\phi(t) \in L_{2}(T, T+h)$ и оценим скалярное произведение $\left(F_{N}(t), \phi(t)\right)$ в пространстве $L_{2}(T, T+h)$ :

$$
\left|\left(F_{N}, \phi\right)\right| \leqslant \sum_{n=-N}^{N}\left\|\int_{l_{n}} \tilde{f}(\lambda) \psi(\lambda) d \lambda\right\|,
$$

где $\psi(\lambda)=\int_{T}^{T+h} e^{\lambda t} \phi(t) d t$. Применяя два раза неравенство Коши-Буняковского, имеем

$$
\begin{aligned}
\left|\left(F_{N}, \phi\right)\right| & \leqslant \sum_{n=-N}^{N}\|\tilde{f}(\lambda)\|_{L_{2}\left(l_{n}\right)}\|\psi(\lambda)\|_{L_{2}\left(l_{n}\right)} \\
& \leqslant\left(\sum_{n=-N}^{N}\|\tilde{f}(\lambda)\|_{L_{2}\left(l_{n}\right)}^{2}\right)^{2}\left(\sum_{n=-N}^{N}\|\psi(\lambda)\|_{L_{2}\left(l_{n}\right)}^{2}\right)^{2} .
\end{aligned}
$$

Оценим последнюю сумму. Имеем

$$
\begin{aligned}
\sum_{n=-N}^{N}\|\psi(\lambda)\|_{L_{2}\left(l_{n}\right)}^{2} & =\int_{a-\delta}^{a+\delta} \sum_{n=-N}^{N}\left|\int_{T}^{T+h} e^{i d_{n} t} e^{x t} \phi(t) d t\right|^{2} d x \\
& \leqslant \int_{a-\delta}^{a+\delta} C\left\|e^{x t} \phi(t)\right\|_{L_{2}(T, T+h)}^{2} d x \leqslant C e^{2(a+\delta) T}\|\phi(t)\|_{L_{2}(T, T+h)}^{2}
\end{aligned}
$$

равномерно по $N$, где постоянная $C$ не зависит от $\phi(t)$. Здесь в предпоследнем неравенстве использована лемма 2 из [16]. Аналогично получаем

$$
\sum_{n=-N}^{N}\|\tilde{f}(\lambda)\|_{L_{2}\left(l_{n}\right)}^{2} \leqslant\left. C\left\|\left.u_{0}^{(j)}(\tau)\right|_{L_{2}(0, h)} ^{2} \leqslant C\right\| u_{0}(\tau)\right|_{W_{2}^{m}(0, h)} ^{2} .
$$

Итак, имеем равномерную по $N$ и $\theta$ оценку

$$
\left|\left(F_{N}, \phi\right)\right| \leqslant C e^{(a+\delta) T}\left\|u_{0}(\tau)\right\|_{W_{2}^{m}(0, h)}\|\phi(t)\|_{L_{2}(T, T+h)},
$$

где постоянная $C$ не зависит от $\phi(t)$. Отсюда получаем оценку

$$
\left\|F_{N}\right\|_{L_{2}(T, T+h)} \leqslant C e^{(a+\delta) T}\left\|u_{0}(\tau)\right\|_{W_{2}^{m}(0, h)}
$$

и завершаем доказательство леммы.

Оценим теперь интегралы по горизонтальным отрезкам $\tau_{n}$.

Лемма 5. Для всех $T>0$ интегралы $\int_{\tau_{n}} U(t, \lambda) d \lambda$ стремятся $к$ нулю по норме пространства $W_{2}^{m}(T, T+h)$ при $n \rightarrow \infty$. 
ДокАЗАтЕЛьство. Повторяя рассуждения из доказательства леммы 4, можно показать, что сходится ряд

$$
\sum_{n \in \mathbb{Z}}\left\|\int_{\tau_{n}} U(t, \lambda) d \lambda\right\|_{W_{2}^{m}(T, T+h)} .
$$

Отсюда получаем требуемое утверждение.

Прежде чем оценивать интегралы по $\partial G_{k}$, сформулируем несколько вспомогательных утверждений. Следующая лемма является тривиальным следствием леммы 2 из [16] (см. также лемму 3.2 из [13]).

Лемма 6. Пусть последовательность комплекснъх чисел $\left\{\lambda_{k}\right\}$ лежит в некоторой вертикальной полосе $\left\{\operatorname{Re} \lambda \in\left(C_{1}, C_{2}\right)\right\}$. Причем число членов последовательности, лежащих в прямоугольнике $\left\{\operatorname{Re} \lambda \in\left(C_{1}, C_{2}\right), \operatorname{Im} \lambda \in\left(C_{3}, C_{3}+h\right)\right\}$, ограничено сверху постоянной, не зависящей от $C_{3}$, а зависящей только от высоты прямоугольника $h$. Тогда система функиий $f_{k}(x)=e^{\lambda_{k} x}$ является бесселевой в $L_{2}(0,1)$, т.е. для любой функции $g(x) \in L_{2}(0,1)$ сходится ряд $\sum_{k}\left|\left(g(x), f_{k}(x)\right)\right|^{2}$.

Следующее утверждение является аналогом леммы 3.3 из [13].

ЛЕмма 7. Пусть бункция $R(\lambda)$ аналитична в некоторой области, лежащей в некоторой вертикальной полосе $\left\{\operatorname{Re} \lambda \in\left(C_{1}, C_{2}\right)\right\}$, за исключением полюсов, удовлетворяющих условиям леммы 6. Тогда в-окрестность полюсов для достаточно малого числа є представляется в виде обгединения непересекающихся компонент $G_{k}$, в каждой из которых количество полюсов с учетом кратности есть $q_{k}+1$. Причем $q_{k}+1 \leqslant q$ для некоторого числа $q$ и всех $k$. Пусть $\lambda_{k}^{0}, \ldots, \lambda_{k}^{q_{k}}-$ полюса $R(\lambda)$, лежащие в $G_{k}$, и вне $\varepsilon$-окрестности этих полюсов для функиии $R(\lambda)$ и ее производных выполняется оценка

$$
\left|R^{(j)}(\lambda)\right|<C, \quad j=0, \ldots, q-1 .
$$

Тогда при $T>0$ справедливо представление

$$
\int_{\partial G_{k}} e^{\lambda(T+x)} R(\lambda) \int_{0}^{1} f(\xi) e^{-\lambda \xi} d \xi d \lambda=T^{q-1} e^{\varkappa T} c_{k} \varphi_{k}(x, T),
$$

где $\varkappa=\sup _{k} \max _{j=0, \ldots, q_{k}} \operatorname{Re} \lambda_{k}^{j}$, последовательность чисел $\left\{c_{k}\right\}$ принадлежит пространству $l_{2}$, а система функиий $\left\{\varphi_{k}(x, T)\right\}$ бесселева по $x$ в $L_{2}(0,1)$, т.е. для всех $T>0$ и для любой функции $g(x) \in L_{2}(0,1)$ сходится ряд $\sum_{k}\left|\left(g(x), \varphi_{k}(x, T)\right)\right|^{2}$, причем сходимость равномерна по $T>0$.

ДокАЗАтЕЛЬСтво. Рассмотрим сначала случай, когда все полюсы простые. Введем функцию

$$
\mathscr{F}_{k}(x, \xi, T)=T^{-q} e^{-\varkappa T} \int_{\partial G_{k}} R(\lambda) e^{\lambda T} e^{\lambda(x-\xi)} d \lambda
$$

и запишем ее в виде

$$
\begin{aligned}
\mathscr{F}_{k}(x, \xi, T) & =T^{-q+1} e^{-\varkappa T} \sum_{j=0}^{q_{k}} a_{k j} e^{\lambda_{k}^{j} T} e^{\lambda_{k}^{j}(x-\xi)} \\
& =T^{-q+1} e^{-\varkappa T} e^{\rho_{k}(x-\xi)} \sum_{j=0}^{q_{k}} a_{k j} e^{\lambda_{k}^{j} T} e^{\left(\lambda_{k}^{j}-\rho_{k}\right)(x-\xi)},
\end{aligned}
$$


где $a_{k j}=\lim _{\lambda \rightarrow \lambda_{k}^{j}} R(\lambda)\left(\lambda-\lambda_{k}^{j}\right)$ и выбрана некоторая точка $\rho_{k} \in G_{k}$ так, что $\operatorname{Re} \rho_{k} \leqslant \varkappa$. Далее, разлагая экспоненты в ряд, имеем

$$
\mathscr{F}_{k}(x, \xi, T)=T^{-q+1} e^{-\varkappa T} e^{\rho_{k}(x-\xi)} \sum_{\mu, \nu=0}^{\infty} \sum_{j=0}^{q_{k}} a_{k j} e^{\lambda_{k}^{j} T}\left(\lambda_{k}^{j}-\rho_{k}\right)^{\mu+\nu} \frac{x^{\mu}(-\xi)^{\nu}}{\mu ! \nu !} ;
$$

тогда

$$
\left(\mathscr{F}_{k}(x, \xi, T), \bar{f}(\xi)\right)=e^{\rho_{k} x} \sum_{\mu=0}^{\infty} \frac{d^{\mu} x^{\mu}}{\mu !} b_{k \mu}(T),
$$

где

$$
\begin{aligned}
b_{k \mu}(T) & =\sum_{\nu=0}^{\infty} b_{k \mu \nu}(T), \\
b_{k \mu \nu}(T) & =T^{-q+1} e^{-\varkappa T} d^{-\mu} \sum_{j=0}^{q_{k}} a_{k j} e^{\lambda_{k}^{j} T}\left(\lambda_{k}^{j}-\rho_{k}\right)^{\mu+\nu} \frac{(-1)^{\nu}}{\nu !}\left(e^{-\rho_{k} \xi}, \xi^{\nu} \bar{f}(\xi)\right) .
\end{aligned}
$$

Оценим $b_{k \mu \nu}(T)$. В каждой области $G_{k}$ представим функцию $R(\lambda)$ в виде

$$
R(\lambda)=\frac{R_{k}(\lambda)}{\prod_{j=0}^{q_{k}}\left(\lambda-\lambda_{k}^{j}\right)},
$$

где $R_{k}(\lambda)$ аналитична в области $G_{k}$ и некоторой ее окрестности. Причем, в виду $(27)$ и принципа максимума эта функция и ее производные $R_{k}^{(l)}(\lambda)$ ограничены в области $G_{k}$ при $l=0, \ldots, q_{k}$ равномерно по $k$. Тогда

$$
\sum_{j=0}^{q_{k}} a_{k j} e^{\lambda_{k}^{j} T}\left(\lambda_{k}^{j}-\rho_{k}\right)^{\mu+\nu}=\sum_{j=0}^{q_{k}} \frac{H_{k}\left(\lambda_{k}^{j}\right)}{\prod_{l \neq j}\left(\lambda_{k}^{j}-\lambda_{k}^{l}\right)}=H_{k}\left(\lambda_{k}^{0}, \ldots, \lambda_{k}^{q_{k}}\right),
$$

где

$$
H_{k}(\lambda)=R_{k}(\lambda)\left(\lambda-\rho_{k}\right)^{\mu+\nu} e^{\lambda T},
$$

а $H_{k}\left(\lambda_{k}^{0}, \ldots, \lambda_{k}^{q_{k}}\right)$ - разделенная разность, соответствующая функции $H_{k}(\lambda)$ и точкам $\lambda_{k}^{0}, \ldots, \lambda_{k}^{q_{k}}$ (см. $[17 ;$ гл. $4, \S 24$, п. 4 , с. 228$]$ ). Известна оценка (см. там же)

$$
\left|H_{k}\left(\lambda_{k}^{0}, \ldots, \lambda_{k}^{q_{k}}\right)\right| \leqslant \frac{1}{q_{k} !} \max _{\lambda \in Q_{k}}\left|H_{k}^{\left(q_{k}\right)}(\lambda)\right|,
$$

где $Q_{k}$ - выпуклая оболочка точек $\lambda_{k}^{0}, \ldots, \lambda_{k}^{q_{k}}$. Без ограничения общности в $\varepsilon$-окрестности $Q_{k}$ нет других полюсов $R(\lambda)$, кроме $\lambda_{k}^{0}, \ldots, \lambda_{k}^{q_{k}}$. Тогда имеем оценку

$$
\begin{aligned}
\max _{\lambda \in Q_{k}}\left|H_{k}^{\left(q_{k}\right)}(\lambda)\right| & =\max _{\lambda \in Q_{k}}\left|\sum_{\alpha+\beta+\gamma=q_{k}} R_{k}^{(\alpha)}(\lambda) \frac{d^{\beta}}{d \lambda^{\beta}}\left(\left(\lambda-\rho_{k}\right)^{\mu+\nu}\right) T^{\gamma} e^{\lambda T}\right| \\
& \leqslant C(\mu+\nu)^{q} d^{\mu+\nu} T^{q-1} e^{\varkappa T}
\end{aligned}
$$

где $d=\max \left(1, \sup _{k} \operatorname{diam} G_{k}\right)$.

Таким образом, получаем, что

$$
\left|b_{k \mu \nu}(T)\right| \leqslant C \frac{d^{\mu}(\mu+\nu)^{q-1}}{\nu !}\left|\left(e^{-\rho_{k} \xi}, \xi^{\nu} \bar{f}(\xi)\right)\right|=c_{k \nu}
$$


равномерно по $T$. Применяя лемму 6 , получаем

$$
\sum_{\nu=0}^{\infty} \sum_{k=1}^{\infty}\left|2^{\nu} c_{k \nu}\right|^{2} \leqslant \sum_{\nu=0}^{\infty} \frac{4^{\nu} d^{\nu}}{\nu !}\left\|\xi^{\nu} f\right\|^{2} \leqslant C\|f\|^{2} .
$$

Обозначая $c_{k}^{\prime}=\sup _{\nu \in[0,+\infty)} 2^{\nu} c_{k \nu}$, имеем

$$
\sum_{k=1}^{\infty}\left|c_{k}^{\prime}\right| \leqslant\|f\|^{2}
$$

Положим $c_{k}=\sum_{\nu=0}^{\infty} c_{k} \nu$. Тогда

$$
\left|b_{k \mu}(T)\right| \leqslant c_{k}
$$

и

$$
\sum_{k=1}^{\infty}\left|c_{k}\right|^{2}=\sum_{k=1}^{\infty}\left|\sum_{\nu=0}^{\infty} c_{k \nu}\right|^{2} \leqslant \sum_{k=1}^{\infty}\left|c_{k}^{\prime}\right|^{2}\left(\sum_{\nu=0}^{\infty} 2^{-\nu}\right)^{2} \leqslant 4 C\|f\|^{2},
$$

т.е. числа $c_{k}$ образуют последовательность из $l_{2}$.

Запишем (29) в виде

$$
\left(\mathscr{F}_{k}(x, \xi, T), \bar{f}(\xi)\right)=c_{k} \varphi_{k}(x, T)
$$

где

$$
\varphi_{k}(x, T)=e^{\rho_{k} x} \sum_{\mu=0}^{\infty} \frac{d^{\mu}}{\mu !} x^{\mu} \frac{b_{k \mu}(T)}{c_{k}} .
$$

Пользуясь $(31)$ и леммой 6 , для любой функции $g(t) \in L_{2}(0,1)$ получаем оценку

$$
\sum_{k=1}^{\infty}\left|\left(\varphi_{k}, g\right)\right|^{2} \leqslant \sum_{k=1}^{\infty} \sum_{\mu=0}^{\infty}\left|\left(e^{\rho_{k} x}, \frac{C_{1}^{\mu}}{\mu !} x^{\mu} g\right)\right|^{2} \leqslant C_{2}\|g\|^{2}
$$

равномерную по $T$, т.е. функции $\varphi_{k}(x, T)$ образуют бесселеву систему по $x$.

Случай кратных полюсов рассматривается как предельный случай простых полюсов дословным повторением рассуждений из [13].

Оценим, наконец, интегралы по контурам $\partial G_{k}$.

ЛЕмма 8. При достаточно больших $T>0$ справедлива оценка

$$
\left\|\sum_{k=1}^{\infty} \int_{\partial G_{k}} U(t, \lambda) d \lambda\right\|_{W_{2}^{m}(T, T+h)} \leqslant C T^{q-1} e^{\varkappa T}\left\|u_{0}(t)\right\|_{W_{2}^{m}(0, h)},
$$

где постоянная $C$ не зависит от начальной функции $u_{0}(t)$.

ДоКАЗАтЕЛЬСтво. Запишем производную порядка $l=0,1, \ldots, m$ от частичной суммы интегралов по $\partial G_{k}$ в виде

$$
\frac{d^{l}}{d t^{l}} \sum_{k=1}^{n} \int_{\partial G_{k}} U(t, \lambda) d \lambda=\sum_{j=0}^{m} \int_{0}^{h} J_{n}\left(u_{0}^{(j)}, t-\theta\right) d \sigma_{j}(\theta),
$$


где

$$
J_{n}(f, x)=\sum_{k=1}^{n} \int_{\partial G_{k}} e^{\lambda x} \lambda^{l} \Delta^{-1}(\lambda) \int_{0}^{h} f(\tau) e^{-\lambda \tau} d \tau d \lambda .
$$

Оценим норму $J_{n}(f, x)$ как функции от $x$ в пространстве $L_{2}(T, T+h)$. Введем обозначение

$$
\mathscr{F}_{k}(x, \tau)=e^{-\varkappa T} \int_{\partial G_{k}} e^{\lambda(T+x-\tau)} \lambda^{l} \Delta^{-1}(\lambda) d \lambda
$$

и запишем (34) в виде

$$
J_{n}(f, T+x) e^{-\varkappa T}=\sum_{k=1}^{n}\left(\mathscr{F}_{k}(x, \tau), f(\tau)\right) .
$$

Применим лемму 7 для $R(\lambda)=\lambda^{l} \Delta^{-1}(\lambda)$ и области Г. Получаем

$$
\left(\mathscr{F}_{k}(x, \tau), f(\tau)\right)=T^{q-1} c_{k} \varphi_{k}(x, T)
$$

где $\left\{c_{k}\right\} \in l_{2}$, а система функций $\left\{\varphi_{k}(x, T)\right\}$ бесселева по $x$ в $L_{2}(0, h)$, причем для всех $g(x) \in L_{2}(0, h)$ ряд $\sum_{k}\left|\left(g(x), \varphi_{k}(x, T)\right)\right|^{2}$ сходится равномерно по $T$. Тогда, обозначая

$$
F_{n}(x, \tau)=\sum_{k=1}^{n} \mathscr{F}_{k}(x, \tau)
$$

и применяя неравенство Коши-Буняковского, имеем для всех $g(x) \in L_{2}(0, h)$ равномерную по $n$ оценку

$$
\begin{aligned}
\left|\left(F_{n}(x, \tau), f(\tau) g(x)\right)_{L_{2}(0, h)^{2}}\right| & =\left|\left(\sum_{k=1}^{n} T^{q-1} c_{k} \varphi_{k}(x, T), g(x)\right)\right| \\
& \leqslant T^{q-1} \sum_{k=1}^{n}\left|c_{k}\right|\left|\left(\varphi_{k}(x, T), g(x)\right)\right| \\
& \leqslant T^{q-1}\left\|c_{k}\right\|_{l_{2}}\left\|\left(\varphi_{k}(x, T), g(x)\right)\right\|_{l_{2}} .
\end{aligned}
$$

Так как функции вида $f(\tau) g(x)$ плотны в $L_{2}(0, h)^{2}$, то по теореме Банаха-Штейнгауза (см. [15]) получаем, что $\left\|F_{n}(x, \tau)\right\|_{L_{2}(0, h)^{2}} \leqslant C T^{q-1}$ равномерно по $n$. Тогда

$$
\left\|J_{n}(f, T+x) e^{-\varkappa T}\right\|_{L_{2}(0, h)}=\left\|\left(F_{n}(x, \tau), f(\tau)\right)\right\| \leqslant C T^{q-1}\|f\| .
$$

Итак, имеем равномерную по $n$ оценку

$$
\left\|J_{n}(f, x)\right\|_{L_{2}(T, T+h)} \leqslant C T^{q-1} e^{\varkappa T}\|f\| .
$$

Теперь функция (33) оценивается достаточно просто. Для каждого слагаемого в (33) имеем

$$
\left\|\int_{0}^{h} J_{n}\left(u^{(j)}, t-\theta\right) d \sigma_{j}(\theta)\right\|_{L_{2}(T, T+h)}^{2}=\int_{T}^{T+h}\left|\int_{0}^{h} J_{n}\left(u^{(j)}, t-\theta\right) d \sigma_{j}(\theta)\right|^{2} d t .
$$


Раскрывая модуль в последнем выражении, меняя порядок интегрирования и используя оценку (35), получаем равномерную по $n$ оценку

$$
\left\|\int_{0}^{h} J_{n}\left(u^{(j)}, t-\theta\right) d \sigma_{j}(\theta)\right\|_{L_{2}(T, T+h)} \leqslant C T^{q-1} e^{\varkappa T}\left\|u_{0}^{(j)}\right\|_{L_{2}(0, h)},
$$

откуда следует искомая оценка (32).

ДоКАЗАТЕЛЬСтво тЕОРЕмы 2. Оценка (9) следует из представления (24), равенства $(25)$ и оценок из лемм 4,5 и 8.

Рассмотрим теперь случай, когда начальная функция $u_{0}(t)$ не удовлетворяет условию (22). Тогда оценка (9) может быть получена следующим образом. Оценка решения $u(t)$ в пространстве $L_{2}(T, T+h)$ получается применением техники, описанной выше. Оценим производную $u^{\prime}(t)$ в пространстве $L_{2}(T, T+h)$. Положим

$$
p_{j}^{1}(\lambda, \theta)=p_{j}(\lambda, \theta)-e^{-\lambda \theta} \lambda^{j-1} u(0), \quad j=0,1, \ldots, m .
$$

Тогда преобразование Лапласа производной $u^{\prime}(t)$ имеет вид

$$
\begin{aligned}
\int_{0}^{\infty} u^{\prime}(t) e^{-\lambda t} d t & =\lambda \int_{0}^{\infty} u(t) e^{-\lambda t} d t-u(0) \\
& =\lambda \Delta^{-1}(\lambda) \sum_{j=0}^{m} \int_{0}^{h}\left(e^{-\lambda \theta} \lambda^{j-1} u(0)+p_{j}^{1}(s, \theta)\right) d \sigma_{j}(\theta)-u(0) \\
& =\lambda \Delta^{-1}(\lambda)\left(\frac{\Delta(\lambda)}{\lambda} u(0)+\sum_{j=0}^{m} \int_{0}^{h} p_{j}^{1}(\lambda, \theta) d \sigma_{j}(\theta)\right)-u(0) \\
& =\lambda^{m} \Delta^{-1}(\lambda) \sum_{j=0}^{m} \int_{0}^{h} \frac{p_{j}^{1}(\lambda, \theta)}{\lambda^{m-1}} d \sigma_{j}(\theta)
\end{aligned}
$$

Заметим, что на любой вертикальной прямой

$$
\frac{p_{j}^{1}(\lambda, \theta)}{\lambda^{m-1}}=O\left(\frac{1}{\lambda}\right) \quad \text { при } \quad \lambda \rightarrow \infty,
$$

и поэтому можно применить использованную выше технику и оценить норму функции $u^{\prime}(t)$ в пространстве $L_{2}(T, T+h)$.

Аналогично, положим

$$
p_{j}^{2}(\lambda, \theta)=p_{j}^{1}(\lambda, \theta)-e^{-\lambda \theta} \lambda^{j-2} u^{\prime}(0), \quad j=0,1, \ldots, m .
$$

Тогда преобразование Лапласа второй производной $u^{\prime \prime}(t)$ имеет вид

$$
\int_{0}^{\infty} u^{\prime \prime}(t) e^{-\lambda t} d t=\lambda \int_{0}^{\infty} u^{\prime}(t) e^{-\lambda t} d t-u^{\prime}(0)=\lambda^{m} \Delta^{-1}(\lambda) \sum_{j=0}^{m} \int_{0}^{h} \frac{p_{j}^{2}(\lambda, \theta)}{\lambda^{m-2}} d \sigma_{j}(\theta) .
$$

Как и выше, на любой вертикальной прямой имеем

$$
\frac{p_{j}^{2}(\lambda, \theta)}{\lambda^{m-2}}=O\left(\frac{1}{\lambda}\right) \quad \text { при } \quad \lambda \rightarrow \infty,
$$

откуда получаем оценку функции $u^{\prime \prime}(t)$. Аналогично оцениваются и остальные производные $u^{(k)}, k=1, \ldots, m$, т.е. норма решения $u(t)$ в пространстве $W_{2}^{m}(T, T+h)$. Теорема доказана. 
Сделаем в заключение следующее замечание. Введем в пространстве $W_{2}^{m}(-h, 0)$ полугруппу $U_{t}$ сдвигов вдоль решений

$$
\left(U_{t} u_{0}\right)(\tau)=u(t+\tau), \quad t \geqslant 0, \quad \tau \in[-h, 0],
$$

где $u(t)$ - решение задачи $(5),(6)$ с начальной функцией $u_{0}(t)$. Можно показать (см., например, [4], [8]), что операторы $U_{t}$ образуют $C_{0}$-полугруппу, генератор $A$ которой имеет вид

$$
\begin{aligned}
A u & =u^{\prime}, \\
D(A) & =\left\{u(t) \in W_{2}^{m+1}(-h, 0): \sum_{j=0}^{m} \int_{0}^{h} u^{(j)}(-\theta) d \sigma_{j}(\theta)=0\right\} .
\end{aligned}
$$

Собственные значения (с.з.) и собственные и присоединенные функции (с.п.ф.) оператора $A$ совпадают с с.з. и с.п.ф. следующей краевой задачи с параметром в граничных условиях:

$$
\begin{aligned}
l(y, \lambda) & =y^{\prime}-\lambda y=0, \\
U(y, \lambda) & =\sum_{j=0}^{m} \int_{0}^{h} \lambda^{j} y(-\theta) d \sigma_{j}(\theta)=0 .
\end{aligned}
$$

Для случая, когда функции $\sigma_{j}(\theta)$ кусочно-постоянные с конечным числом скачков, в работе [13] построены в различных пространствах операторы, линеаризующие эту краевую задачу, и показывается, что их с.п.ф. образуют базис Рисса со скобками в соответствующих функциональных пространствах. В частности, с.п.ф. оператора $A$ в этом случае образуют базис Рисса со скобками в пространстве $W_{2}^{m}(-h, 0)$. Тогда оценка (9) решений задачи (5), (6) следует из теоремы 1 работы [18].

Краевые задачи для обыкновенных дифференциальных уравнений с интегральными краевыми условиями, не содержащими параметр, изучались в [19]. Пользуясь методами этих двух работ [19] и [13], а также леммами 1 и 7 настоящей работы, можно построить в широком классе функциональных пространств операторы, линеаризующие краевую задачу (36), (37), и показать, что их с.п.ф., отвечающие с.з., попавшим в полосу П, образуют базис Рисса со скобками в замыкании своей линейной оболочки. Причем в скобки нужно объединять лишь те слагаемые, которые соответствуют с.з., попавшим в одну и ту же область $G_{k}$. В частности, с.п.ф. оператора $A$ образуют базис Рисса со скобками в замыкании своей линейной оболочки в пространстве $W_{2}^{m}(-h, 0)$. Тогда решение $u(t)$ может быть представлено в виде $u(t)=v(t)+w(t)$, где основной вклад в рост решения дает функция $v(t)$ - проекция решения $u(t)$ на замыкание линейной оболочки с.п.ф., соответствующих с.з., попавшим в полосу П. Эта функция $v(t)$, а, значит, и решение $u(t)$, оценивается опять с помощью теоремы 1 работы [18].

Автор искренне благодарит проф. А. А. Шкаликова за постановку задачи и ценные советы.

\section{СПИСОК ЦИТИРОВАННОЙ ЛИТЕРАТУРЫ}

[1] O. Diekmann, S. A. van Gils, S. M. Verduyn Lunel, H.-O. Walther, Delay Equations. Functional-, Complex-, and Nonlinear Analysis, Springer-Verlag, Berlin, 1995. 
[2] Р. Беллман, К. Кук, Дифференциально-разностные уравнения, Мир, М., 1967.

[3] А. Д. Мышкис, Линейные дифференииальные уравнения с запаздываюоим аргументом, Наука, М., 1972.

[4] Дж. Хейл, Теория функиионально-дифференциальных уравнений, Мир, М., 1984.

[5] Л.Э. Эльсгольц, Введение в теорию дифференииальных уравнений с отклоняющимся аргументом, Наука, М., 1964.

[6] D. Henry, "Linear autonomous neutral functional differential equations", J. Differential Equations, 15 (1974), 106-128.

[7] В. В. Власов, С. А. Иванов, "О точных оценках решений дифференциально-разностных уравнений нейтрального типа", Докл. РАН, 406:5 (2006), 583-585.

[8] V.V. Vlasov, D. A. Medvedev, "On asymptotic behavior and estimates of solutions to neutral equations", Funct. Differential Equations, 13:2 (2006), 207-223.

[9] Б. Я. Левин, Распределение корней целых функиий, Гостехиздат, М., 1956.

[10] Л.С. Понтрягин, "О нулях некоторых элементарных трансцендентных функций", Изв. АН СССР, Сер. матем., 6:3 (1942), 115-134.

[11] В. А. Садовничий, В. А. Любишкин, Ю. Белабасси, "О нулях целых функций одного класса", Тр. сем. им. И. Г. Петровского, 8 (1982), 211-217.

[12] А.А. Шкаликов, "Теоремы тауберова типа о распределении нулей голоморфных функций”, Матем. сб., 123:3 (1984), 317-347.

[13] А.А. Шкаликов, "Краевые задачи для обыкновенных дифференциальных уравнений с параметром в граничных условиях", Тр. сем. им. И.Г. Петровского, 9 (1983), 190-229.

[14] В.В. Власов, С. А. Иванов, "Оценки решений уравнений с последействием в шкале пространств Соболева и базис из разделенных разностей", Алгебра и анализ, 15:4 (2003), 115-141.

[15] А. Н. Колмогоров, С. В. Фомин, Элементы теории функиий и функиионалъного анализа, Наука, М., 1989.

[16] В.Э. Кацнельсон, “О базисах из показательных функций в $L^{2}$ ”, Функцион. анализ и его прилож., 5:1 (1971), 37-47.

[17] Г. Е. Шилов, Математический анализ. Второй специалъный курс, Наука, М., 1965.

[18] А.И. Милославский, "Об устойчивости некоторых классов эволюционных уравнений”, Сиб. матем. журн., 26:5 (1985), 118-132.

[19] А.А. Шкаликов, "О базисности собственных функций обыкновенных дифференциальных операторов с интегральными краевыми условиями", Вестн. МГУ. Сер. 1. Матем., мех., 1982, №6, 12-21.

А. А. Лесных

Московский государственный университет

им. М. В. Ломоносова

E-mail: andrey_les@mail.ru
Поступило

20.11.2006 